\title{
Towards an entropy-based detached-eddy simulation
}

\author{
ZHAO Rui ${ }^{*}$, YAN Chao ${ }^{1}$, LI XinLiang ${ }^{2} \&$ KONG WeiXuan ${ }^{1}$ \\ ${ }^{1}$ National Laboratory for Computational Fluid Dynamics, Beijing University of Aeronautics and Astronautics, Beijing 100191, China; \\ ${ }^{2}$ Key Laboratory of High Temperature Gas Dynamics, Institute of Mechanics, Chinese Academy of Sciences, Beijing 100190, China
}

Received March 22, 2012; accepted October 23, 2012

\begin{abstract}
A concept of entropy increment ratio $(\bar{s})$ is introduced for compressible turbulence simulation through a series of direct numerical simulations (DNS). $\bar{s}$ represents the dissipation rate per unit mechanical energy with the benefit of independence of freestream Mach numbers. Based on this feature, we construct the shielding function $f_{\mathrm{s}}$ to describe the boundary layer region and propose an entropy-based detached-eddy simulation method (SDES). This approach follows the spirit of delayed detached-eddy simulation (DDES) proposed by Spalart et al. in 2005, but it exhibits much better behavior after their performances are compared in the following flows, namely, pure attached flow with thick boundary layer (a supersonic flat-plate flow with high Reynolds number), fully separated flow (the supersonic base flow), and separated-reattached flow (the supersonic cavity-ramp flow). The Reynolds-averaged Navier-Stokes (RANS) resolved region is reliably preserved and the modeled stress depletion (MSD) phenomenon which is inherent in DES and DDES is partly alleviated. Moreover, this new hybrid strategy is simple and general, making it applicable to other models related to the boundary layer predictions.
\end{abstract}

hybrid RANS/LES, detached-eddy simulation, turbulence, compressible flows, entropy

PACS number(s): 47.27.E2, 47.32.Ff, 47.40.-x, 65.40.Gr

Citation: Zhao R, Yan C, Li X L, et al. Towards an entropy-based detached-eddy simulation. Sci China-Phys Mech Astron, 2013, 56: 1970-1980, doi: $10.1007 / \mathrm{s} 11433-012-4976-2$

Advances in computer speeds have made it possible to utilize more accurate methods of simulating and modeling turbulent flows. However, grid requirements and time cost typically restrict direct numerical simulation (DNS) and even large eddy simulation (LES) to only low Reynolds numbers. That is, Reynolds-averaged Navier-Stokes (RANS) equations along with a turbulence model seem still to be a powerful tool for engineering aerodynamic analysis nowadays, whereas, traditional RANS approaches do not resolve any turbulent flow structures, but model the effect of turbulence on the mean flow in terms of representative mean turbulence scales. As a result, all spectral effects are lost in the time averaging process. The unsteady variant of this, URANS, although managing to resolve non-stationary mean flows, will produce too much eddy viscosity polluting the

*Corresponding author (email: zhaorui@ase.buaa.edu.cn) predicted fields [1,2]. For many turbulent flows of engineering importance, traditional RANS or URANS modeling may be an awkward approach or may fail to reproduce the relevant flow physics.

Hybrid RANS/LES approaches [3] represent a credible alternative improving the description of such flows at a reasonable cost by taking into account most of the flow unsteadiness. The main idea of these methods is to model the turbulent structures in the attached region of the flow and to solve the large length-scale structures elsewhere. One of the most popular RANS/LES methods is the detached-eddy simulation proposed by Spalart et al. in 1997 [4] (termed in the following as DES97), which is based on a modification of the length scale employed by Spalart-Allmaras RANS model (SA) [5]. Since the time it was put forward, DES97 has been applied successfully to numerous engineering flow problems, especially in high-Reynolds number separated 
flows by a sizeable community. However, DES97 does require strict rules for grid generation to obtain a consistent solution [6], since the hybrid interface directly depends on the grid spacing. Of particular concern during grid generation is that the grid may exceed a critical level of refinement, e.g. excessive grid refinements in streamwise and spanwise direction can make the interface move towards the inner boundary layer, disregarding the actual flow structure. Part of the RANS region will be switched to LES and then the underlying modeled Reynolds stresses are not sufficiently provided, leading to modeled stress depletion (MSD) and even non-physical separation [7]. To avoid such problems, Spalart et al. [8] constructed the delayed detached-eddy simulation (DDES) to "preserve RANS mode," or "delay LES function" in the region where an attached turbulence boundary layer is expected. The main idea of DDES is utilizing the parameter $r_{\mathrm{d}}$ to detect the boundary layer, which is concluded to be robust and effective with detailed comparisons through several test cases. However, recent studies $[9,10]$ have found that the MSD problem is still inherited by DDES, even the improved version IDDES approach which is designed to function as wall-modeled LES (WMLES) near the wall [11]. One explanation is that the grid setting in the outer boundary layer region is too coarse to compensate the lacking modeled Reynolds stresses by directly resolving turbulent fluctuation. As the shielding function $f_{\mathrm{d}}\left(f_{\mathrm{dt}}\right.$ in IDDES) itself depends on the decreasing eddy viscosity, the reserved boundary layer range is further diminished in a self-amplifying process. As a result, even after considerable simulation time, the separation keeps on growing and a pre-mature separation may occur [10].

On the other hand, in the near wall region, as for the fierce turbulent fluctuation and wall frication, a portion of mechanical energy is irreversibly transformed into internal energy, i.e. the entropy increases. Through previous DNS investigations of the supersonic compression ramp flow, we found that the value of entropy could discern the boundary layer region, while it keeps monotonously decreasing normal to the wall [12]. In the present study, we try to enlarge the capability of entropy and propose an entropy-based shielding function to reliably reserve the boundary layer. Focusing on this ambition, this paper is organized as follows.

\section{Entropy increment ratio}

Virtually all flows of practical engineering interest are turbulent. Due to the irregular motion of turbulence, the transportations of mass, momentum and energy are enhanced, while the extra energy is dissipated. In the near wall region, as a result of the fierce turbulent fluctuation and wall frication, a portion of mechanical energy is irreversibly transformed into internal energy, i.e. the entropy increases. A similar process also occurs in the deceleration of flows where shock waves appear. In this research, we define the entropy function as that in ref. [12]:

$$
s=\frac{p}{\rho^{r}}
$$

where $p$ and $\rho$ are the local pressure and density respectively, and $r=1.4$ is the specific heat ratio. In particular, this function is derived with the perfect gas assumption, denoting the local rate of energy dissipation for compressible flows. When the external potential flows pass the body surface, the velocity is gradually decreased to zero due to the viscous frication. Naturally, the values of entropy near the wall are directly related to inflow speeds, i.e. the entropy increment becomes comparatively small with a low inflow speed. In order to get rid of this dependence on freestream Mach numbers, the entropy increment ratio $(\bar{s})$ is introduced as below:

$$
\bar{s}=\frac{s / s_{\infty}-1}{M a_{\infty}^{2}} .
$$

Here, the subscript $\infty$ means the freestream values.

$\bar{s}$ represents the dissipation rate per unit mechanical energy. We extract the corresponding quantities from DNS results for further evaluation. Table 1 shows the simulation conditions of DNS [13-15]. The incoming flows are subsonic, supersonic and hypersonic, respectively. As aforementioned, the values of $s$ near the wall show great disparities, varying about one order of magnitude from $M a=0.7$ to $M a=6$ (Figure 1(a)). On the other hand, $\bar{s}$ exhibits a consistent behavior depicted in Figure 1(b). The values near the wall are all falling into the range of $0.1-0.5$, unrelated to the freestream Mach numbers. This character is convenient as we always expect a model or a physical parameter to be applicable for different flow conditions. Additionally, the range where $\bar{s}>0$ is almost equal to the extent where $u / U_{\infty}<1.0$, which means that $\bar{s}$ could be used to discern the boundary layer region of these simple flows.

Table 2 lists the simulation conditions of DNS for the supersonic compression ramp flow. We extract the profiles of $\bar{s}$ at the locations ahead and among the separation bubble, which is also the shock/boundary-layer interaction area (Figure 2(a)). Once more, the values near the wall are also in the range of $0.1-0.5$ (Figure 2(b)). Comparing the distributions of streamwise velocity with the values of $\bar{s}$ (Figure

Table 1 Imulation conditions for boundary layer flows ${ }^{\text {a) }}$

\begin{tabular}{cccc}
\hline & $M a_{\infty}$ & $R e /$ in & $T_{\mathrm{w}} / T_{\infty}$ \\
\hline Case1 & 0.7 & $5.0 \times 10^{4}$ & 1.098 \\
Case2 & 2.25 & $6.35 \times 10^{5}$ & 1.9 \\
Case3 & 6 & $2.0 \times 10^{6}$ & 6.98 \\
\hline
\end{tabular}

a) $M a_{\infty}$ : inflow Mach number; Re/in: Reynolds number per inch; $T_{\mathrm{w}}$ : wall temperature; $T_{\infty}$ : inflow temperature 

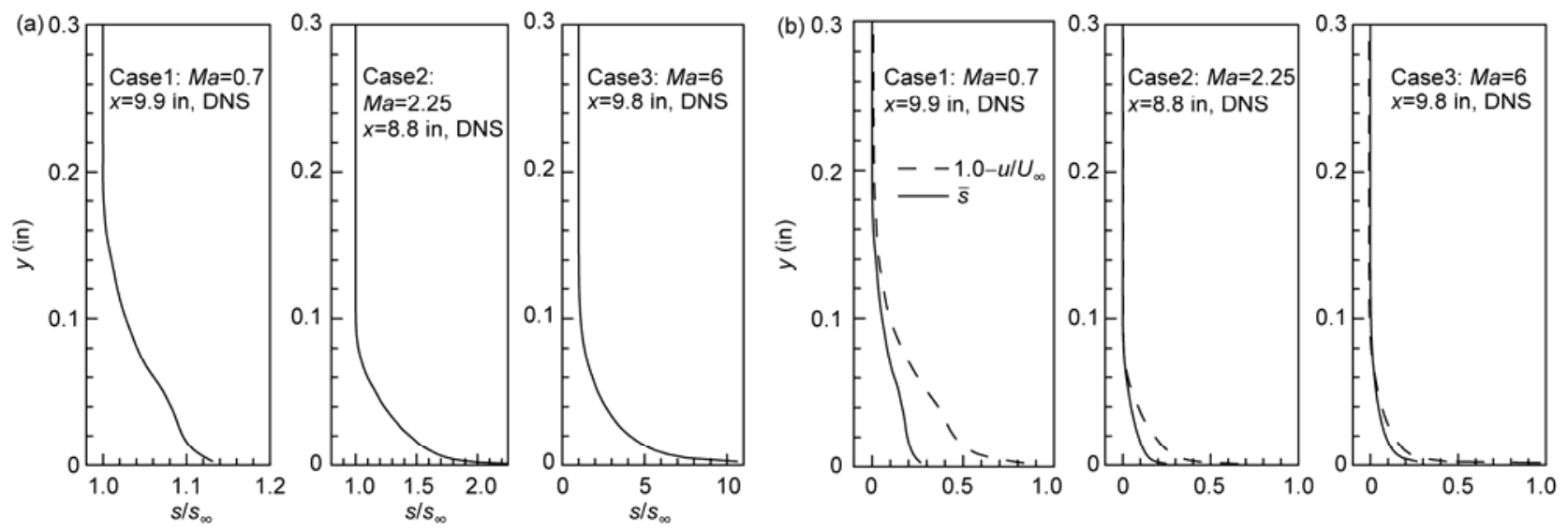

Figure 1 Entropy and entropy increment ratio profiles normal to the wall of different Mach numbers. (a) Entropy profiles normal to the wall; (b) entropy increment ratio (solid line) and velocity (dashed line) profiles.

Table 2 Simulation conditions for the incoming turbulent boundary layer (at $x=-30 \mathrm{~mm})^{\text {a) }}$

\begin{tabular}{cccccc}
\hline$M a_{\infty}$ & $R e_{\theta}$ & $\theta(\mathrm{mm})$ & $\delta(\mathrm{mm})$ & $T_{\infty}(\mathrm{K})$ & \\
\hline 2.9 & 2344 & 0.42 & 6.5 & 108.1 & 307 \\
\hline
\end{tabular}

a) $R e_{\theta}$ : Reynolds number based on momentum thickness; $\theta$ : momentum thickness; $\delta$ : boundary layer thickness


Figure 2 Entropy increment ratio profiles normal to the wall at different locations. (a) Entropy contour of the compression ramp (DNS); (b) entropy increment ratio (solid line) and velocity (dashed line) profiles.

2(b)), $\bar{s}$ could denote the boundary layer range where $\bar{s}>$ 0 in this complex flow.

\section{Numerical method}

Since the derivation of the entropy concept is based on the perfect gas assumption for compressible flows, the governing equations describing the mean flowfield are the timedependent, compressible Reynolds-averaged Navier-Stokes equations. Other restrictions on the equations include the constant specific heats and the Sutherland viscosity law. To get rid of the numerical dissipation as far as possible, we use the $5^{\text {th }}$ order WENO scheme [16] for the inviscid terms combined with $4^{\text {th }}$ order central differencing [17] for the viscous terms is employed. Second-order accuracy is obtained in the temporal discretization via dual-time stepping with sub-iterative procedure. By removing the transient shedding cycles (about initial 30 cycles), statistics are in general compiled over periods of at least 50 vortex shedding cycles to reach full convergence.

\subsection{Spalart-Allmaras model}

The one-equation model of Spalart-Allmaras (SA) [5] is chosen as the base for the construction of DES97, DDES and SDES. The transport equation for a variable $\hat{v}$ related to the eddy viscosity reads 


$$
\begin{aligned}
\frac{\partial \hat{v}}{\partial t}+u_{j} \frac{\partial \hat{v}}{\partial x_{j}}= & C_{b 1}\left[1-f_{t 2}\right] \Omega \hat{v} \\
& +\left\{C_{b 1}\left[\left(1-f_{t 2}\right) f_{v 2}+f_{t 2}\right] \frac{1}{\kappa^{2}}-C_{w 1} f_{w}\right\}\left(\frac{\hat{v}}{\tilde{d}}\right)^{2} \\
& +\frac{1}{\sigma} \frac{\partial}{\partial x_{j}}\left[\left(v+\left(1+C_{b 2}\right) \hat{v}\right) \frac{\partial \hat{v}}{\partial x_{j}}\right]-\frac{C_{b 2}}{\sigma} \hat{v} \frac{\partial^{2} \hat{v}}{\partial x_{j}^{2}}
\end{aligned}
$$

where $\Omega=\sqrt{2 W_{i j} W_{i j}}$ is the measure of vorticity, and the length scale $\tilde{d}=d_{\mathrm{w}}$ is actually the distance to the nearest wall. Definitions for the remaining variables can be found in the previously cited references. The first term on the right hand is the production term, the second term is the destruction term, and the rest are the diffusion terms.

\subsection{Detached-eddy simulation}

The DES97 method relies on a new length scale $\tilde{d}$ in replacement of $d_{\mathrm{w}}$ given by

$$
\tilde{d}=\min \left(d_{\mathrm{w}}, C_{\mathrm{DES}} \Delta\right),
$$

where $C_{\mathrm{DES}}$ is a calibrated constant equal to 0.65 , and $\Delta$ is the grid spacing defined by $\Delta=\max (\Delta x, \Delta y, \Delta z)$.

As emphasized by Travin et al. [18], DES97 uses a single turbulence model which functions as a subgrid scale model in detached flows and a Reynolds-averaged model in attached flow regions. Away from the wall, the turbulence production and destruction should roughly balance each other. Under this assumption, it is easy to conclude from eq. (3) that the SA model is reduced to the familiar Smagorinsky's subgrid scale model [19].

$$
\begin{gathered}
\tilde{v} \approx \Delta^{2} \Omega, \text { DES } 97, \\
v_{\mathrm{SGS}} \approx \Delta^{2} S, \text { Smagorinsky, }
\end{gathered}
$$

where $S=\sqrt{2 S_{i j} S_{i j}}$ is the measurement of the strain tensor.

To be consistent with the following DDES and SDES expressions, eq. (4) is rewritten as below:

$$
\tilde{d}=d_{\mathrm{w}}-f \max \left(0, d_{\mathrm{w}}-C_{\mathrm{DES}} \Delta\right),
$$

where

$$
f= \begin{cases}0, & d_{\mathrm{w}}<C_{\mathrm{DES}} \Delta, \\ 1, & d_{\mathrm{w}} \geqslant C_{\mathrm{DES}} \Delta .\end{cases}
$$

That is, when $d_{\mathrm{w}}<C_{\mathrm{DES}} \Delta, \tilde{d}=d_{\mathrm{w}}$ and the simulation operates in RANS mode. On the contrary, when $d_{\mathrm{w}} \geqslant$ $C_{\mathrm{DES}} \Delta$, the model exhibits the LES behavior. Moreover, the subgrid model behaves somewhat like a dynamic model because of the material derivative and the diffusion term.
Nevertheless, and from the beginning, a special concern is devoted to the region, named "gray-area", where the model switches from RANS to LES. In particular, a grid-induced premature switch to LES mode may occur in the attached part of the flow, resulting in a loss of modeled turbulent stresses. In order to get rid of this drawback, Spalart et al. [8] proposed DDES with a modification of $\tilde{d}$ to delay this switch, and then prevented the MSD problem. The formation of DDES is presented in the following context.

\subsection{Delayed detached-eddy simulation}

DDES is designed to ensure that the attached boundary layers are treated in RANS regardless of the grid resolution by using the quantity:

$$
r_{\mathrm{d}}=\frac{v_{\mathrm{t}}+v}{\sqrt{U_{i, j} U_{i, j}} \kappa^{2} d_{\mathrm{w}}^{2}},
$$

where $v_{\mathrm{t}}$ is the kinematic eddy viscosity, $v$ is the molecular viscosity, $U_{i, j}$ are the velocity gradients, $\kappa=0.41$ is the von Karman's constant, and $d_{\mathrm{w}}$ is the distance to the wall.

According to ref. [8], the parameter $r_{\mathrm{d}}$ is slightly modified relative to the $\mathrm{SA}$ definition. The value of $r_{\mathrm{d}}$ equals 1.0 in the logarithmic layer, and falls to 0 gradually towards the edge of the boundary layer and is used in the function:

$$
f_{\mathrm{d}}=1-\tanh \left(\left[8 r_{\mathrm{d}}\right]^{3}\right) \text {, }
$$

which equals 0 in the boundary layer and 1.0 elsewhere.

The length scale $\tilde{d}$ used in DES97 is then modified to be a function of $f_{\mathrm{d}}$ and is defined by

$$
\tilde{d}=d_{\mathrm{w}}-f_{\mathrm{d}} \max \left(0, d_{\mathrm{w}}-C_{\mathrm{DES}} \Delta\right) .
$$

As its literal meaning, the function $f_{\mathrm{d}}$ should delay the premature switch to the LES mode and ensure that the boundary layer is fully treated in the RANS mode. However, according to Spalart et al. [11], the MSD problem is still inherited by DDES. They further introduced the IDDES approach for WMLES application, but the above flaw is not completely eliminated since eq. (8) still serves as a part of the improved formula [11]. This inherent deficiency prompts the other "delayed" strategy below.

\subsection{Entropy-based detached-eddy simulation}

A new version of detached-eddy simulation based on the concept of entropy increment ratio (named SDES) is presented in this paper. It follows the "delayed" spirit of DDES, but from an energy dissipation aspect.

Taking advantage of the proposed concept $\bar{s}$, we construct the parameter $r_{\mathrm{s}}$ relative to the DDES definition:

$$
r_{\mathrm{s}}=\frac{\bar{s}}{l_{\mathrm{s}}^{2}}
$$


where $l_{\mathrm{S}}$ is the subgrid length-scale designed to be less than 1.0 in the boundary layer and increases rapidly in the LES region. We utilize the ratio of the corresponding length scales of RANS and LES by constructing $l_{\mathrm{s}}$ as below:

$$
l_{\mathrm{s}}=C_{\mathrm{g}} \kappa d_{\mathrm{w}} / C_{\mathrm{DES}} \Delta
$$

in which $C_{\mathrm{g}}=3 \sqrt[3]{\Delta_{x} \Delta_{y} \Delta_{z}} /\left(\Delta_{x}+\Delta_{y}+\Delta_{z}\right)$ is the grid deformation ratio, and $C_{\mathrm{DES}}=0.65$ is the Smagorinsky constant. $C_{\mathrm{g}}$ is less than 1.0 in the anisotropy grids near the wall and around 1.0 in the typical LES region. The combination of $\kappa d_{\mathrm{w}}$ represents the Prandtl-van Driest RANS length scale, while $C_{\mathrm{DES}} \Delta$ is the LES subgrid scale employed by DES97 and DDES.

The quantity $r_{\mathrm{s}}$ is used in the same function of eq. (8) with no more constants:

$$
f_{\mathrm{s}}=1.0-\tanh \left(r_{\mathrm{s}}\right),
$$

$f_{\mathrm{d}}$ in eq. (9) is then substituted by $f_{\mathrm{s}}$ and the length scale $\tilde{d}$ is modified by

$$
\tilde{d}=d_{\mathrm{w}}-f_{\mathrm{s}} \max \left(0, d_{\mathrm{w}}-C_{\mathrm{DES}} \Delta\right) .
$$

This new hybrid strategy does not represent a minor adjustment within DES-like framework; there is a qualitative change. First, the value of $\bar{s}$ included in $r_{\mathrm{s}}$ depends on the local mean flow field rather than the turbulent field adopted by DDES and IDDES. Therefore, the blending function $f_{\mathrm{s}}$ could get rid of the self-amplifying process of $f_{\mathrm{d}}$ mentioned in ref. [10]. In other words, $f_{\mathrm{s}}$ is completely independent of any specific RANS model. Its behavior is only determined by the local energy dissipation and grid resolution. Second, the constants 8 and 3 included in $f_{\mathrm{d}}$, which are based on intuitive shape requirements, are no longer needed. It means that this hybrid method may be more universal.

\section{Results and discussion}

\subsection{Supersonic flat-plate flow}

To evaluate the performance of the newly developed SDES method in attached flows, we simulated the supersonic flow over a flat plate with a freestream $M a=2.25$ and $R e=6.35$ $\times 10^{5}$ /in (Table 1). After our refinement procedure, a threedimensional ambiguous grid was generated to exhibit the MSD problem, as depicted in Figure 3. The grid resolution in the plane parallel to the wall $\left(\Delta x^{+}=100\right.$ and $\left.\Delta z^{+}=100\right)$ ranges between classical values used in LES and RANS. The results of SDES, DES97 and DDES at $x=8.8$ in are extracted and compared with the RANS computation, as shown in Figures 4 and 5.

With this flow condition and extreme grid, it is observed that the superiority of DDES is completely degraded. Both DES97 and DDES underestimate the eddy viscosity by

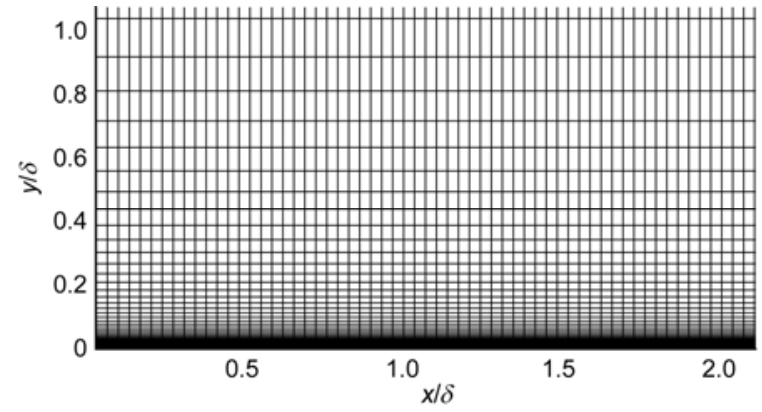

Figure 3 Grid used to show the MSD problem. $\delta$ is the boundary layer thickness at $x=8.8 \mathrm{in}$.



Figure 4 Turbulent viscosity profiles at $x=8.8 \mathrm{in}$.

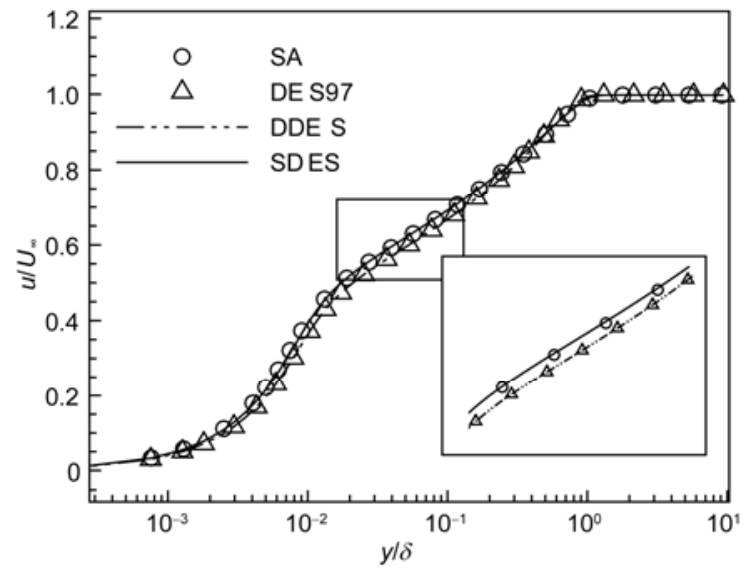

Figure 5 Velocity profiles at $x=8.8$ in

almost $40 \%$ (Figure 4), while the velocity profiles obviously depart from that of SA model at the log-law region (Figure $5)$. This potential deficiency of DDES (IDDES) was reported in ref. [10] by simulating the HGR-01 airfoil at stall, and the reason was concluded to the self-amplifying process, i. e. once the "delayed function" $f_{\mathrm{d}}\left(f_{\mathrm{dt}}\right.$ in IDDES) fails to cover the whole boundary layer, there is a lack of modeled Reynolds stresses since the near-wall grid is not fine enough to directly resolve the turbulent fluctuation. The formula of 
$f_{\mathrm{d}}$ (eq. (7)) itself explicitly depends on the decreasing eddy viscosity, and then the shielded part of the boundary layer is further reduced in a self-amplifying process. However, through our investigation here (Figure 6), we find that only about $10 \%$ of the boundary layer is covered by evaluating $f_{\mathrm{d}}$ based on the steady SA-RANS and SST-RANS [20] solutions. The reserved region where $f_{\mathrm{d}}<1.0$ is obviously smaller than the area where $d_{\mathrm{w}}<C_{\mathrm{DES}} \Delta$, which means that the shielding function $f_{\mathrm{d}}$ has already lost the "delayed" ability at the beginning of time advancing. As a consequence, the DDES results are completely consistent with that of DES97. On the other hand, since the entropy increment ratio $\bar{s}$ could accurately denote the boundary layer (Figure 1(b)), and its value is independent of freestream Mach numbers, the hybrid function $f_{\mathrm{s}}$ could more reliably reserve the RANS resolved region. Thus, the results of SDES agree well with that of SA model.

\subsection{Supersonic base flow}

This test case is aimed to verify the capability of SDES in fully separated flows, which are considered to be the primary application of DES-like methods. The supersonic flow passes a cylindrical sting of radius $R=31.75 \mathrm{~mm}$, experimentally investigated by Herrin and Dutton [21]. The two-dimensional slice of the time-averaged flowfield is shown in Figure 7. The flow is separated from the sharp corner, turning through an expansion fan before recompressing downstream of the recirculation zone. Due to the recompression, a shock wave is formed. The experimental freestream conditions are given in Table 3.

The length of the cylinder is extended to $8 R$, which is the same as that used in refs. [22,23] to match the upstream boundary layer thickness at a distance of $1 \mathrm{~mm}$ prior to the base. Figure 8 depicts the construction of the gird, which consists $2.39 \times 10^{6}$ cells and has been proved fine enough to represent the flowfield [24]. The gird is clustered near the wall and the spacing is increased in a proper ratio. The

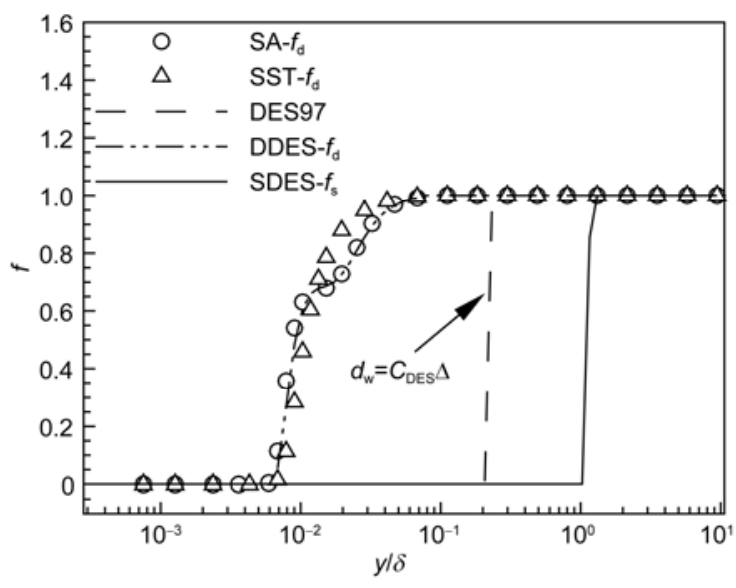

Figure 6 Function $f$ profiles at $x=8.8$ in. $f=0$ : RANS controlled areas.

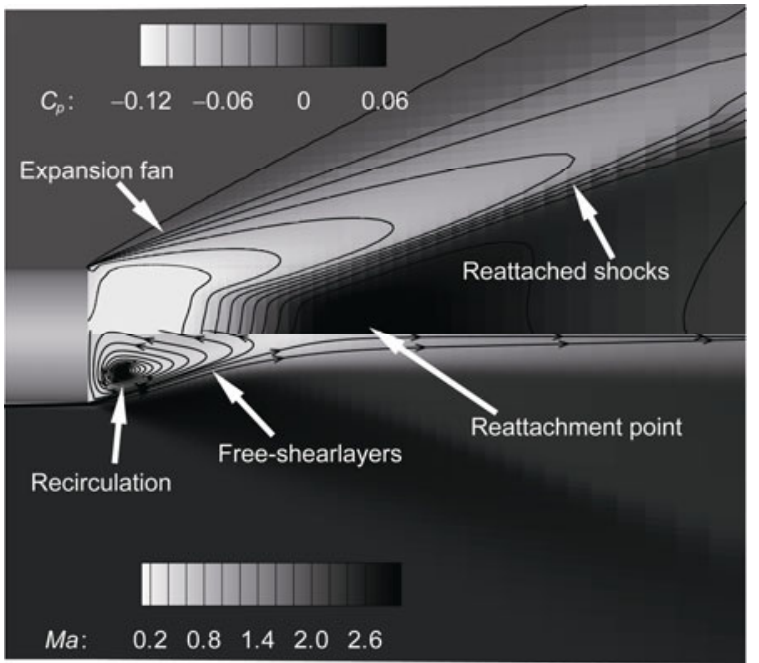

Figure 7 Schematic diagram of the time-averaged axisymmetric base flow.

Table 3 Flow conditions for the supersonic axisymmetric base flow

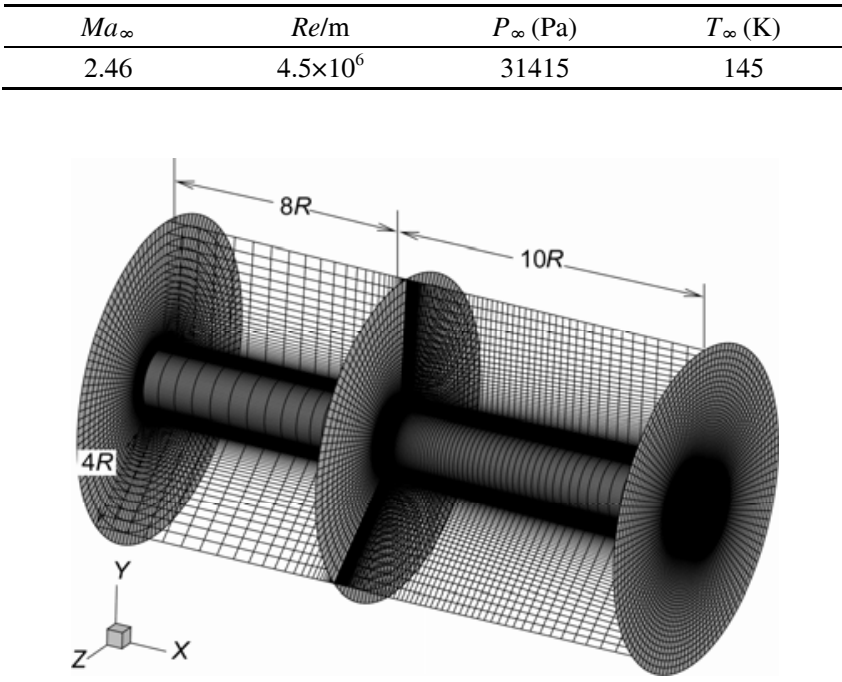

Figure 8 Grid construction for the base flow.

distance of the first gird line to the wall is $10^{-5}$ which corresponds to a $y^{+}$less than 1.0. No-slip boundary condition is employed at the adiabatic surface.

Figure 9 compares the instantaneous contours of the function $f$ around the surface, calculated by each hybrid strategy. Since the hybrid interface of DES97 completely depends on the grid construction and the grid is refined at the corner, the RANS modeled region is gradually diminishing in the streamwise direction (Figure 9(a)). For this supersonic flow, $f_{\mathrm{d}}$ used in DDES preserved so little RANS modeled region along the sting, which again confirms the potential deficiencies revealed in sect. 3.1 (Figure 9(b)). Meanwhile, the shielding function $f_{\mathrm{s}}$ employed by SDES indicates a reasonable boundary layer before the corner but preserved a larger RANS modeled region at the base 

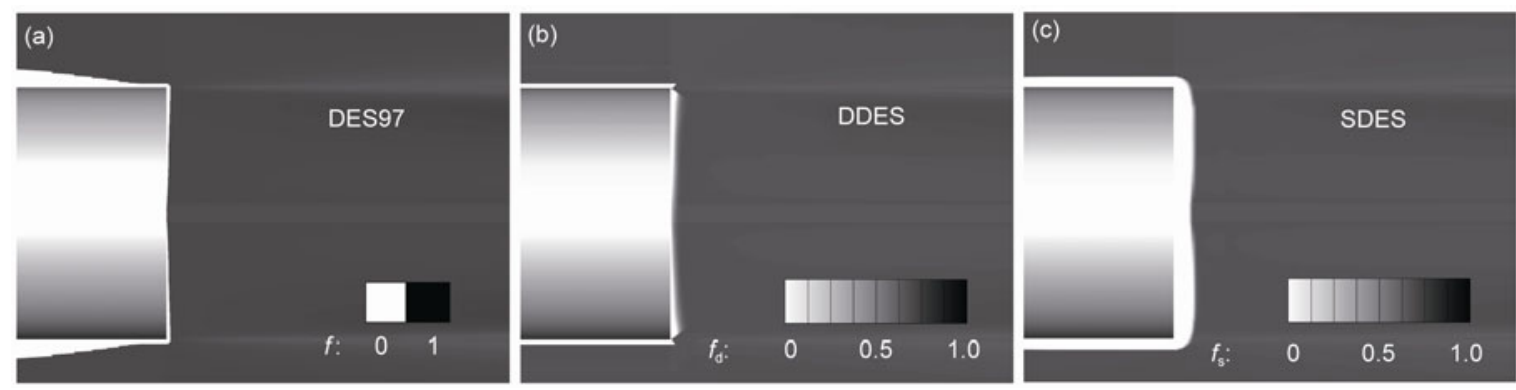

Figure 9 Instantaneous contours of function $f . f=0$ : RANS controlled areas. (a) DES97 result; (b) DDES result; (c) SDES result.

(Figure 9(c)). The influences of these different hybrid interfaces are discussed in the later sections.

The comparisons of velocity and eddy viscosity profiles on the cylinder lateral surface $1 \mathrm{~mm}$ upstream of the corner are shown in Figures 10 and 11, respectively. Although all the hybrid methods predict the boundary layer velocity reasonably well, there are slight discrepancies among the eddy viscosity distributions under this grid resolution. As depicted in Figure 9(a), since the hybrid interface moves down to

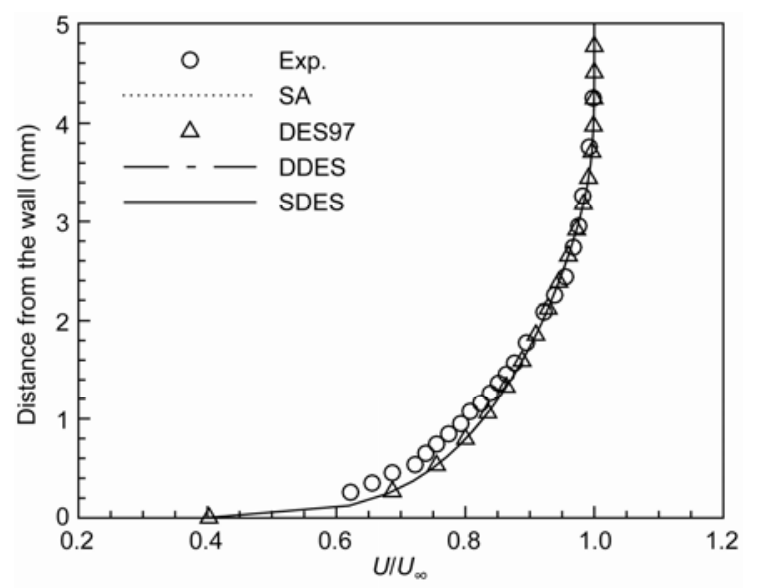

Figure 10 Velocity profiles at $x=-1 \mathrm{~mm}$.

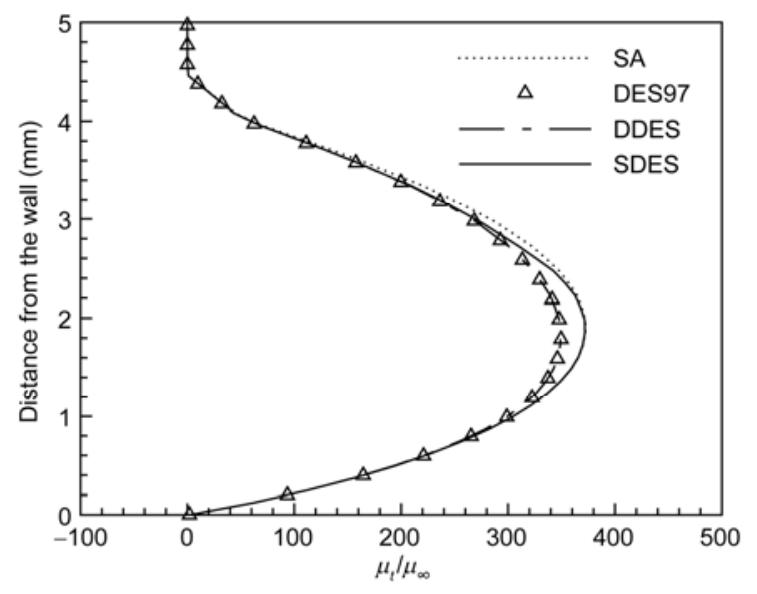

Figure 11 Turbulent viscosity profiles at $x=-1 \mathrm{~mm}$. the inner boundary layer because of the grid refinement at the corner, the eddy viscosity is not produced sufficiently and the MSD problem comes up again. On the other hand, SDES represents the incoming boundary layer properties satisfactorily. Because the performance of DDES is regressed to that of DES97, we only compare the results of other methods in the following.

The time-averaged flowfield calculated by SA, DES97 and SDES, respectively, is compared with the experimental result in Figure 12. Computed axial velocity distributions along the wake axis behind the corner separation are shown in Figure 13. In the RANS computation, the free shear layers from the corner are diffused and curved significantly toward the wake axis compared to the experiment, as depicted in Figure 12(a). The reason is considered to be in the large turbulent eddy viscosity induced by the nature of the turbulence model [25]. The large turbulent eddy viscosity accelerates the free shear layer growth rate, resulting in an underpredicted recirculation zone (Figure 13). As for the DES97 and SDES computations, the local Mach number profiles behind the base agrees quite well with the experiment, as shown in Figures 12(b) and (c) although the recirculation zones are slightly overpredicted (Figure 13). Furthermore, since the entropy-based hybrid function $f_{\mathrm{s}}$ preserved a larger RANS modeled region at the base (Figure 9(c)), the reversed flow is slowed down and the velocity profile is more consistent with the experiment near the base (Figure 13).

Further investigations are undertaken by plotting the streamwise velocity component and primary Reynolds shear stress profiles at locations $x / R=0.1575,0.9449,2.5197$, as depicted in Figures 14 and 15. Due to the excessive turbulent eddy viscosity, SA model fails to reproduce the velocity profiles at all locations. On the other hand, the results of DES97 and SDES are in better agreement with the experiment, as shown in Figure 14. However, discrepancies among the calculated Reynolds shear stress profiles could be observed in Figure 15. At the location $x / R=0.1575$, where the boundary layer has just got separated from the cylinder, both DES97 and SDES could correctly predict the position and the peak of the Reynolds shear stress. Due to the larger RANS modeled region at the base, the turbulent fluctuation is partly suppressed and the profile calculated by SDES is 


\section{(a)}

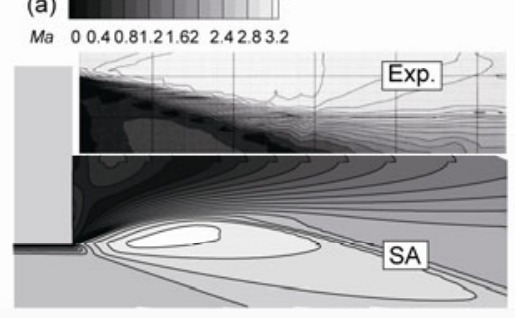

(b)

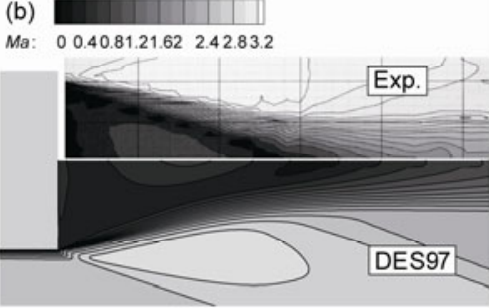

(c)

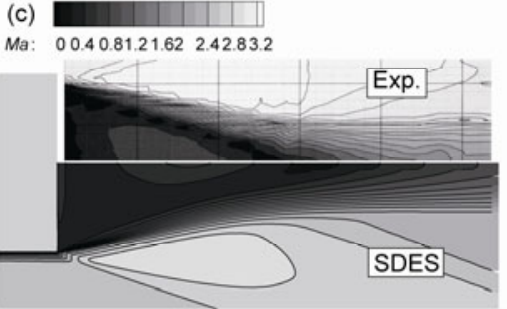

Figure 12 Mach number contours in the wake region. (a) SA result; (b) DES97 result; (c) SDES result.

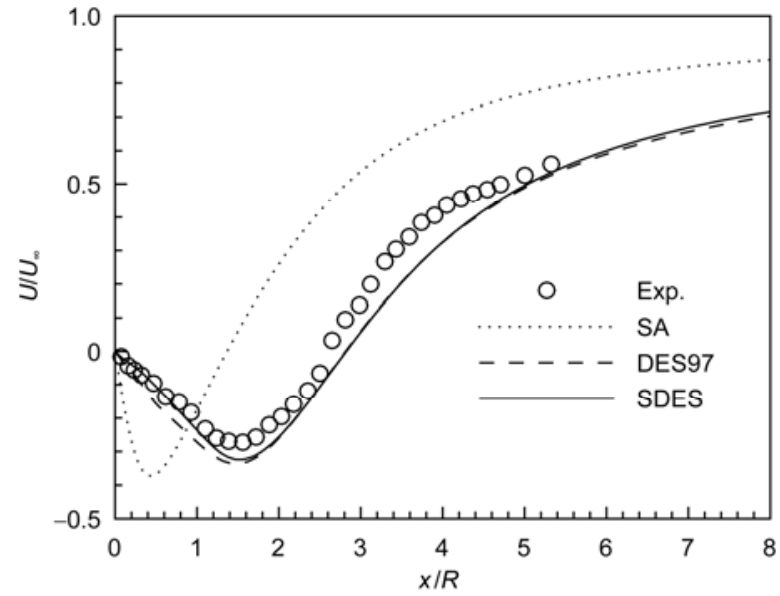

Figure 13 Time-averaged streamwise velocity along the centerline.

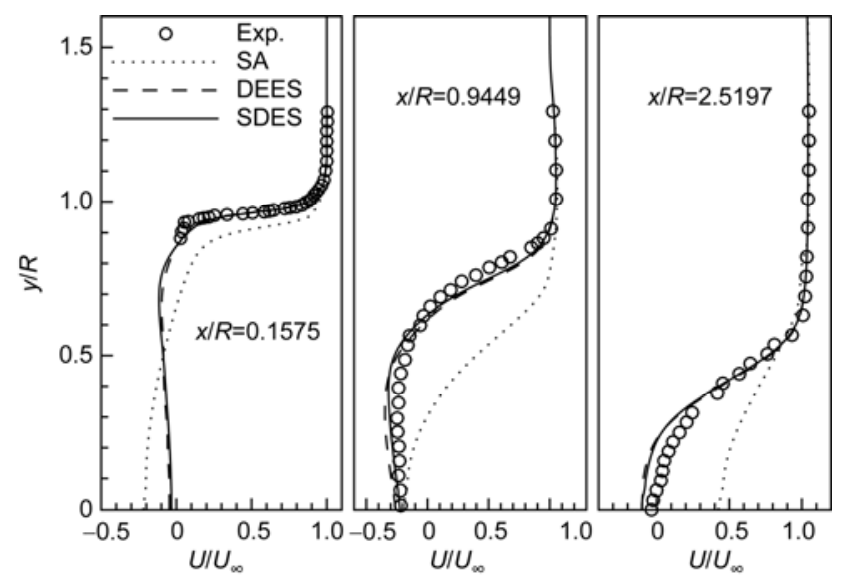

Figure 14 Time-averaged streamwise velocity at different locations.

flatter than that of DES97. At the location $x / R=0.9449$, the shear layer grows under high compressible conditions and the streamlines quickly converge toward the axis. DES97 represents a rounded profile while the result of SDES agrees well with the experiment. Near the reattachment point $x / R=$ 2.5197, DES97 overestimates the peak of Reynolds shear stress but compares well with the experiment near the axis. In all, for this supersonic fully separated flow, both SDES and DES97 are qualified to reproduce the flowfield, while SDES could provide a more accurate incoming boundary

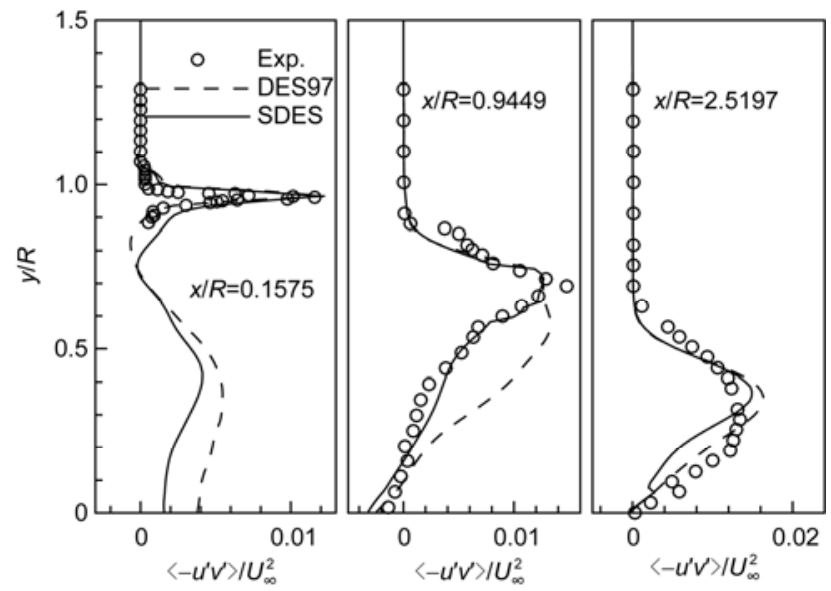

Figure 15 Time-averaged Reynolds shear stress at different locations.

layer.

\subsection{Supersonic cavity-ramp flow}

This flow is a rather severe test for SDES, since in this case it must automatically provide three different types of behavior depending on the flow region. Namely, it should function as a RANS model in the attached boundary layers upstream of the cavity which do not have any "turbulent content", as LES in the separation zone, and, finally, as WMLES in the reattached boundary layer on the ramped portion, which inherits "turbulent content" from the upstream free-shear layer (Figure 16). The supersonic cavity-ramp flowfield was experimentally investigated by Settles et al. [26,27], and detailed test conditions are summarized in Table 4. The inflow conditions are achieved by calculating two-dimensional flow over a flat plate until the boundary layer properties match the experimental values. Periodic boundary conditions are applied in the $Z$ direction, while standard no-slip, adiabatic wall boundary conditions are prescribed along all solid surfaces. Initial condition for the hybrid simulation is obtained by solving the flowfield with SA model.

The methodology of mesh construction is referred to ref. [28]. The $X-Y$ cut of the three-dimensional structured grid is shown in Figure 17. The grid consists of two blocks, containing $37 \times 85 \times 33$ points upstream of the cavity and 154 


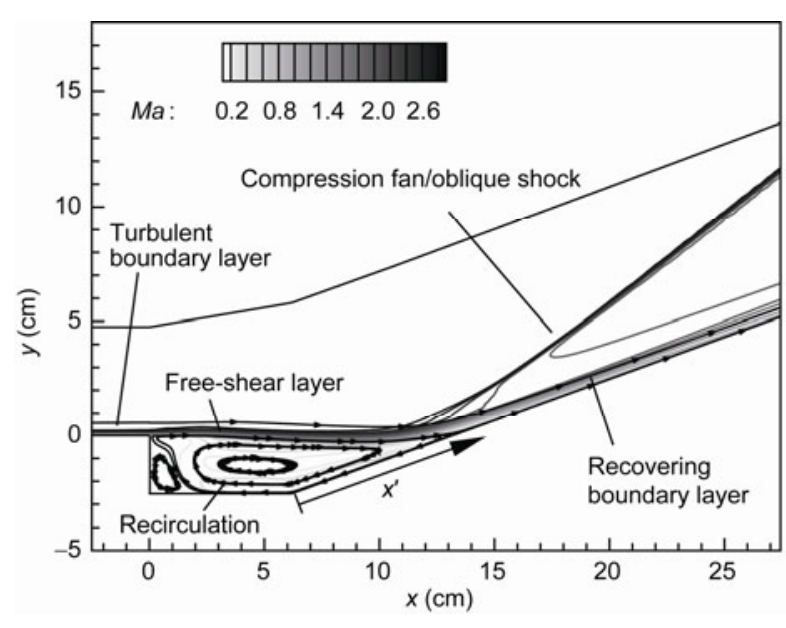

Figure 16 Flow structures for the cavity-ramp (Mach number contour).

Table 4 Conditions for the incoming turbulent boundary layer (at $x=$ $-2.54 \mathrm{~cm})$

\begin{tabular}{ccccc}
\hline$M a_{\infty}$ & $R e / \mathrm{m}$ & $\delta(\mathrm{cm})$ & $P_{\infty}(\mathrm{Pa})$ & $T_{\infty}(\mathrm{K})$ \\
\hline 2.92 & $6.7 \times 10^{7}$ & 0.29 & 21240 & 95.37 \\
\hline
\end{tabular}



Figure 17 Grid construction in the $X-Y$ plane.

$\times 108 \times 33$ points downstream of the leading edge of the cavity. The grid extends $3.81 \mathrm{~cm}$ in the $Z$ direction, which is 1.5 times the cavity height. We clustered the grid to all solid surfaces and paid particular attention to the free-shear layer and reattachment area. It should be noted that the mesh utilized in this study is much too coarse in the spanwise and streamwise directions to be considered suitable for a true LES in the near-wall regions.

Figure 18 shows the snapshots of the function $f$ and the eddy viscosity fields obtained with different hybrid strategies. It seems striking to find that both DES97 and DDES fail to protect a reasonable extent of the boundary layer (Figure 18(a)), resulting in the lacked eddy viscosity distributions (Figure 18(b)). For DES, the grid around the cavity area is refined to capture the free-shear layer structure, so is
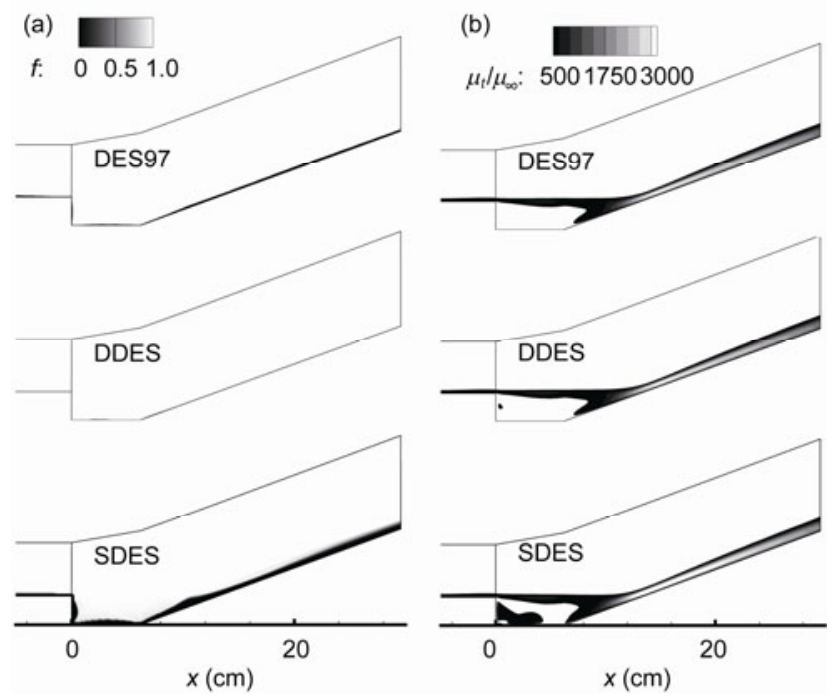

Figure 18 Instantaneous function $f$ and eddy viscosity contours. (a) Function $f$ contour; (b) eddy viscosity contours (only depict $\mu_{t} / \mu_{\infty}>100$ ).

the grid in the ramped portion for the recovering boundary layer. Therefore, the RANS modeled area where $d_{\mathrm{w}}<C_{\mathrm{DES}} \Delta$ becomes inadequate in the above regions. Considering the performance of DDES, the shielding function $f_{\mathrm{d}}$ seems to have lost its effect again and the viscosity distribution is degraded to DES97. In contrast, the proposed shielding function $f_{\mathrm{s}}$ employed in SDES could reliably reserve the boundary layer, resulting in a more prominent level of eddy viscosity.

To ascertain these discrepancies, we show the distributions of $\left(8 r_{\mathrm{d}}\right)^{3}$ and $r_{\mathrm{s}}$ achieved by different models in Figure 19. We present $\left(8 r_{\mathrm{d}}\right)^{3}$ instead of $r_{\mathrm{d}}$ for numerical magnitude consideration. Figures 19(a) and (b) present the distributions of $\left(8 r_{\mathrm{d}}\right)^{3}$ calculated by steady RANS methods, which could be considered the initial flowfield of the unsteady calculation. It is interesting to find that the thickness where $r_{\mathrm{d}}>0.1$ has already been too thin to denote the boundary layer region in the ramped portion, i.e. $r_{\mathrm{d}}$ itself seems not qualified for supersonic flows especially with an adverse pressure gradient since this parameter is almost directly derived from SA model [8]. Compared with the instantaneous results by DDES (Figure 19(c)), the values of $r_{\mathrm{d}}$ are further reduced due to the self-amplifying process mentioned in ref. [10]. These observations indicate that the parameter $r_{\mathrm{d}}$, which has been calibrated for subsonic flows [5], may fail its purpose in applications with more complex flow conditions, e.g. supersonic/hypersonic flows or flows with an adverse pressure gradient. On the other side, the entropy increment ratio $\bar{s}$ is insensitive to the flow conditions, and its value depends on the local mean flow quantities. Based on this advantage, the parameter $r_{\mathrm{s}}$ could give convenient values along the wall (Figure 19(d)) and the proposed shielding function $f_{\mathrm{s}}$ seems to be more "robust" in complex flows.

Figures 20 and 21 present the time-averaged pressure and 

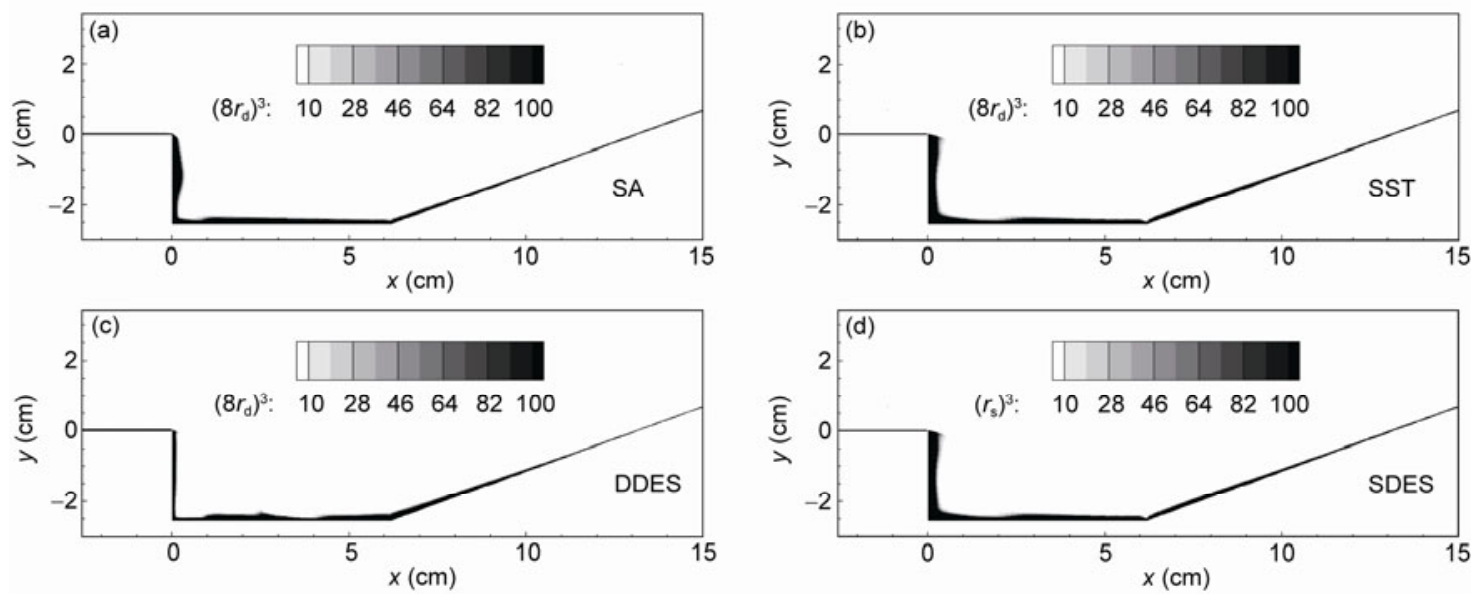

Figure 19 Parameter $r$ contours (only depicting $r>0.1$, for SA, SST and DDES, $r=\left(8 r_{\mathrm{d}}\right)^{3}$; for SDES, $r=r_{\mathrm{s}}$ ). (a) Steady SA result; (b) steady SST result; (c) instantaneous DDES result; (d) instantaneous SDES result.



Figure 20 Time-averaged wall pressure distribution.

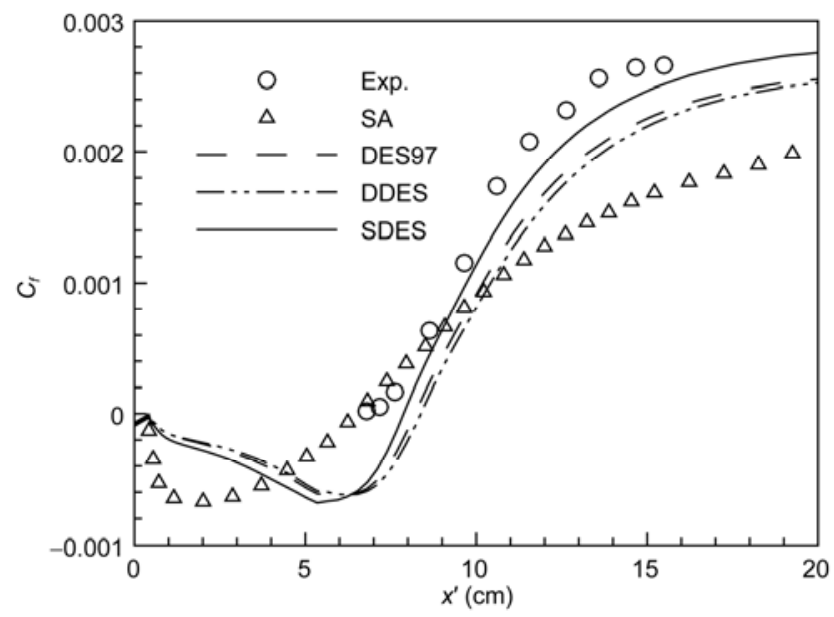

Figure 21 Time-averaged skin frication distribution.

skin friction distributions in the recovery region. Profiles in the recovery region correspond to the distance $x^{\prime}$ along the ramp, measured from the juncture of the cavity and the ramp (Figure 16). Among the three hybrid strategies, the results of SDES are the most consistent with the experimental data. Since the rise of the pressure and skin frication indicates the rate of recovery of the boundary layer downstream of reattachment [28], $f_{\mathrm{s}}$ could indeed denote a comparatively accurate development of the boundary layer. As aforementioned, the friction coefficient distribution is underestimated by DES97 due to the premature switch to LES mode on this grid. The advantage of DDES vanishes and the results accord with that of DES97. Anyhow, all of the three methods show a major priority over the pure RANS model, which generally fails to predict the levels of turbulent fluctuation amplification necessary to produce a rapid recovery.

\section{Conclusions}

A new version of hybrid strategy, named SDES, is proposed from the consideration of energy dissipation for compressible flows. This model performs just as well as DES97 and DDES in massive separated flows, and shows comparative advantage in complex flows with both separated and attached regions. First, a novel concept, named entropy increment ratio $(\bar{s})$, is introduced based on the analysis of DNS data. $\bar{s}$ represents the dissipation rate per unit mechanical energy and its value could distinguish the boundary layer region. Based on this advantage and by including the ratio of turbulent length scales, the shielding function $f_{\mathrm{s}}$ employed by SDES is validated and shown the priority to reliably preserve the RANS modeled region.

Second, the deficiencies of the "delayed function" $f_{\mathrm{d}}$ in DDES $\left(f_{\mathrm{dt}}\right.$ in IDDES) are investigated. On the one hand, the parameter $r_{\mathrm{d}}$ adopted by $f_{\mathrm{d}}$ is directly derived from SA model and calibrated in the subsonic flows. This parameter seems not to be qualified for the supersonic/hypersonic flows, especially with adverse pressure gradients. On the other hand, once the "delayed function" $f_{\mathrm{d}}\left(f_{\mathrm{dt}}\right.$ in IDDES) 
fails to cover the whole boundary layer due to extreme grids or complex flow conditions, the modeled Reynolds stresses are lacking. Since $f_{\mathrm{d}}$ itself explicitly depends on the decreasing eddy viscosity, the shielded part of the boundary layer is further reduced in a self-amplifying process.

Finally, this entropy-based hybrid function $f_{\mathrm{s}}$ is completely independent of any specific RANS model. Its behavior is only determined by the local energy dissipation and grid resolution, while the form is relatively simple. This function may be used to other issues related to the boundary layer, like transition or turbulence modeling. Nevertheless, the derivations of entropy expression for incompressible flows, the hypersonic thermochemical flows, etc., which are all out of the perfect gas assumption, are left for our future investigations.

This work was supported by the National Basic Research Program of China (Grant No. 2009CB724104), the Innovation Foundation of BUAA for PhD Graduates and the Academic New Artist Award of BUAA for PhD Graduates

1 Morton S A, Forsythe J R, Mitchell A M, et al. DES and RANS simulations of delta wing vortical flows. AIAA Paper, 2002, AIAA2002-0587

2 Morton S A, Forsythe J R, Mitchell A M, et al. Detached eddy simulation and Reynolds averaged Navier-Stokes simulations of delta wing vortical flow fields. J Fluids Eng, 2002, 124: 924-932

3 Sagaut P, Deck S, Terracol M. Multiscale and Multiresolution Approaches in Turbulence. London: Imperial College Press, 2006

4 Spalart P R, Jou W H, Allmaras S R. Comments on the feasibility of LES for wings, and on a hybrid RANS/LES approach. In: Proceedings of the 1st AFSOR international Conference on DNS/LES, Greyden Press, Columbus, OH, 1998. 137-147

5 Spalart P R, Allmaras S R. A one-equation turbulence model for aerodynamic flows. AIAA Paper, 1992, AIAA-92-0439

6 Spalart P R. Young person's guide to detached-eddy simulation grids. NASA CR-2001-211032, 2001

7 Spalart P R. Detached-eddy simulation. Annu Rev Fluid Mech, 2009, 41: $181-202$

8 Spalart P R, Deck S, Shur M L, et al. A new version of detached-eddy simulation, resistant to ambiguous grid densities. Theor Comput Fluid Dyn, 2006, 20: 181-195

9 Xing T, Carrica P, Stern F. Large-scale RANS and DDES computations of KVLCC2 at drift angle 0 degree. Gothenburg 2010: A Workshop on CFD in Ship Hydrodynamics, Sweden 2010
10 Axel P, Rolf R, Christoph W, et al. A comparison of detached-eddy simulation and Reynolds-stress modeling applied to the flow over backward-facing step and an airfoil at stall. AIAA paper, 2010, AIAA-2010-920

11 Shur M L, Spalart P R, Strelets M K, et al. A hybrid RANS-LES approach with delayed-DES and wall-modelled LES capabilities. Int J Heat Fluid Fl, 2008, 29: 1638-1649

12 Zhao R, Yan C, Yu J. A new kind Baldwin-Lomax turbulence model under the limit of entropy (in Chinese). J Beijing Univ Aeronaut Astronaut, 2012, 38(2): 175-179

13 Zhou Y, Li X L, Fu D X, et al. Coherent structures in the transition of a flat-plate boundary layer at $M a=0.7$. Chin Phys Lett, 2007, 24(1): $147-150$

14 Li X L, Fu D X, Ma Y W, et al. Acoustic calculation for supersonic turbulent boundary layer flow. Chin Phys Lett, 2009, 26(9): 181-184

15 Li X L, Fu D X, Ma Y W. Direct numerical simulation of a spatially evolving supersonic turbulent boudary layer at $M a=6$. Chin Phys Lett, 2006, 23(6): 1519-1522

16 Nichols R H, Tramel R W, Bunning P G. Evaluation of two high-order weighted essentially nonoscillatory schemes. AIAA J, 2008, 46(12): 3090-3102

17 Shen Y Q, Zha G C, Chen X Y. High order conservative differencing for viscous terms and the application to vortex-induced vibration flows. AIAA Paper, 2008, AIAA-2008-4059

18 Travin A, Shur M, Spalart P R, et al. Detached eddy simulation past a circular cylinder. Flow Turbul Combust, 2000, 63(1): 293-313

19 Smagorinsky J. General circulation experiments with the primitive equations. Mon Weather Rev, 1963, 91(3): 99-164

20 Meter F. Zonal two equation $K-\omega$ turbulence models for aerodynamic flows. AIAA Paper, 1993, AIAA-93-2906

21 Herrin J L, Dutton J C. Supersonic base flow experiments in the near wake of a cylindrical afterbody. AIAA J, 1994, 32(1): 77-83

22 Forsythe J R, Hoffmann K A, Cummings R M, et al. Detached-eddy simulation with compressibility corrections applied to a supersonic axisymmetric base flow. J Fluids Eng, 2002,124: 911-923

23 Peng S H. Algebraic hybrid RANS-LES modeling applied to incompressible and compressible turbulent flows. AIAA paper, 2006, AIAA-2006-3910

24 Xiao Z X, Fu S. Studies of the unsteady supersonic base flows around three afterbodies. Acta Mech Sin, 2009, 25: 471-479

25 Kawai S, Fujii K. Computational study of supersonic base flow using hybrid turbulence methodology. AIAA J, 2005, 43(6): 1265-1275

26 Settles G S, Williams D R, Baca B K, et al. Reattachment of a compressible turbulent free shear layer. AIAA J, 1982, 20(1): 60-67

27 Horstman C C, Settles G S, Williams D R, et al. A reattaching free shear layer in compressible turbulent flow. AIAA J, 1982, 20(1): 7985

28 Fan T C, Tian M, Edwards J R, et al. Validation of a hybrid Reynolds-Averaged/Large-Eddy simulation method for simulating cavity flameholder configurations. AIAA Paper, 2001, AIAA-2001-2929 